Discrete Comput Geom 27:531-550 (2002)

DOI: $10.1007 / \mathrm{s} 00454-001-0083-2$

\title{
A Deterministic Algorithm for Approximating the Mixed Discriminant and Mixed Volume, and a Combinatorial Corollary*
}

\author{
Leonid Gurvits $^{1}$ and Alex Samorodnitsky ${ }^{2}$ \\ ${ }^{1}$ Los Alamos National Laboratory, \\ Los Alamos, NM 87545, USA \\ gurvits@lanl.gov \\ 2 Institute for Advanced Study, \\ Princeton, NJ 08540, USA \\ asamor@ias.edu \\ and \\ DIMACS Center, Rutgers University, \\ Piscataway, NJ 08854-8018, USA
}

\begin{abstract}
We present a deterministic polynomial-time algorithm that computes the mixed discriminant of an $n$-tuple of positive semidefinite matrices to within an exponential multiplicative factor. To this end we extend the notion of doubly stochastic matrix scaling to a larger class of $n$-tuples of positive semidefinite matrices, and provide a polynomial-time algorithm for this scaling. As a corollary, we obtain a deterministic polynomial algorithm that computes the mixed volume of $n$ convex bodies in $\mathbf{R}^{n}$ to within an error which depends only on the dimension. This answers a question of Dyer, Gritzmann and Hufnagel. A "side benefit" is a generalization of Rado's theorem on the existence of a linearly independent transversal.
\end{abstract}

\section{Introduction}

\subsection{Permanent, Mixed Volume and Mixed Discriminant}

Permanent. Let $A=\left(a_{i j}\right)$ be an $n \times n$ matrix. The number

$$
\operatorname{per}(A)=\sum_{\sigma \in S_{n}} \prod_{i=1}^{n} a_{i \sigma(i)},
$$

* Part of this work was done when the first author was at the Institute for Advanced Study, Princeton, and was partially supported by an Alfred P. Sloan Foundation grant. Work by the second author partially supported by a State of New Jersey grant. 
where $S_{n}$ is the symmetric group on $n$ elements, is called the permanent of $A$. For a 0,1 matrix $A, \operatorname{per}(A)$ counts the number of perfect matchings in $G$, the bipartite graph represented by $A$.

It is \# $P$-hard to compute the permanent of a nonnegative (even 0,1 ) matrix [26], and so it is unlikely to be efficiently computable exactly for all matrices. The realistic goal, then, is to try and the permanent efficiently approximate as well as possible, for large classes of matrices.

How well can the permanent be approximated in polynomial time? The first efficient probabilistic algorithm that provides a $2^{O(n)}$-factor approximation for the permanent of a general nonnegative matrix was obtained by Barvinok in [6] and [7].

A deterministic strongly polynomial algorithm also achieving $2^{O(n)}$-factor approximation (with a worse constant in the exponent) for arbitrary nonnegative matrices was constructed in [21]. The algorithm uses matrix scaling to reduce the problem to estimating the permanent of a doubly stochastic matrix. For these matrices the permanent is known to lie in the interval $\left[n ! / n^{n}, 1\right]$, and this solves the approximation problem. We recall that the lower bound of $n ! / n^{n}$ on the permanent of a doubly stochastic matrix was conjectured by van der Waerden and proven by Egorychev [11] and Falikman [12] 50 years later. (A slightly weaker, but sufficient for the purposes of [21], bound of $e^{-n}$ was proven by Friedland [13]).

Recently Jerrum et al. [18] produced an efficient polynomial-time probabilistic algorithm that approximates the permanent extremely tightly $((1+\varepsilon)$-factor $)$, essentially solving the permanent approximation question.

Mixed Volume. Let $K_{1} \cdots K_{n}$ be convex bodies in the Euclidean space $\mathbf{R}^{n}$, and let $V(\cdot)$ be the Euclidean volume in $\mathbf{R}^{n}$. It is well known (see for instance [25]) that the value of $V\left(\lambda_{1} K_{1}+\cdots+\lambda_{n} K_{n}\right)$ is a homogeneous polynomial of degree $n$ in nonnegative variables $\lambda_{1} \cdots \lambda_{n}$, where " + " denotes the Minkowski sum, and $\lambda K$ denotes the dilatation of $K$ with coefficient $\lambda$. The coefficient $V\left(K_{1} \cdots K_{n}\right)$ of $\lambda_{1} \cdot \lambda_{2} \cdot \cdots \cdot \lambda_{n}$ is called the mixed volume of $K_{1} \cdots K_{n}$. Alternatively,

$$
V\left(K_{1} \cdots K_{n}\right)=\frac{\partial^{n}}{\partial \lambda_{1} \cdots \partial \lambda_{n}} V\left(\lambda_{1} K_{1}+\cdots+\lambda_{n} K_{n}\right) .
$$

The mixed volume is known to be monotone [25], namely $K_{i} \subseteq L_{i}$, for $i=1, \ldots, n$, implies $V\left(K_{1} \cdots K_{n}\right) \leq V\left(L_{1} \cdots L_{n}\right)$. In particular, it is always nonnegative.

The problem of computing the mixed volume of convex bodies is important for combinatorics and algebraic geometry [9]. For instance, the number of toric solutions to a generic system of $n$ polynomial equations on $\mathbf{C}^{n}$ is equal to the mixed volume of the Newton polytopes of the equations.

This problem is also \#P-complete, since volume is a special case of mixed volume, and computing the volume is $\# P$-complete ${ }^{1}$ [8]. Therefore, the reasonable goal, once again, is to seek approximate solutions.

\footnotetext{
${ }^{1}$ In fact, as one of the referees has pointed out, mixed volume generalizes permanent. This happens when the bodies $K_{1} \cdots K_{n}$ decompose as sums of $\operatorname{conv}\left(0, a e_{i}\right)$, where $e_{i}$ is the $i$ th standard unit vector.
} 
Efficient polynomial-time probabilistic algorithms that approximate the mixed volume extremely tightly $((1+\varepsilon)$-factor) were developed for some classes of well-presented convex bodies [9].

How well can the mixed volume be approximated in polynomial time? The first efficient probabilistic algorithm that provides an $n^{O(n)}$-factor approximation for arbitrary well-presented proper ${ }^{2}$ convex bodies was obtained by Barvinok in [6].

The question of the existence of an efficient deterministic algorithm for approximating the mixed volume of arbitrary well-presented proper convex bodies with an error depending only on the dimension was posed by Dyer et al. [9]. They quote a lower bound [5] of $(\Omega(n / \log n))^{n / 2}$ for the approximation factor of such an algorithm.

Deterministic polynomial-time algorithms that approximate the mixed volume with a factor of $n^{O(n)}$ were given, for certain classes of proper convex bodies, in [6] and [9].

Mixed Discriminant. Let $A_{1} \cdots A_{n}$ be $n \times n$ real symmetric matrices. It is well known (and easily seen) that the value of $\operatorname{det}\left(x_{1} A_{1}+\cdots+x_{n} A_{n}\right)$ is a homogeneous polynomial of degree $n$ in variables $x_{1} \cdots x_{n}$. The number

$$
D\left(A_{1} \cdots A_{n}\right)=\frac{\partial^{n}}{\partial x_{1} \cdots \partial x_{n}} \operatorname{det}\left(x_{1} A_{1}+\cdots+x_{n} A_{n}\right)
$$

is called the mixed discriminant of $A_{1} \cdots A_{n}$. The mixed discriminant is known [3] to be monotone, namely $A_{i} \preceq B_{i}$, for $i=1, \ldots, n$, implies $D\left(A_{1} \cdots A_{n}\right) \leq D\left(B_{1} \cdots B_{n}\right){ }^{3}$ In particular, if the matrices $A_{1} \cdots A_{n}$ are positive semidefinite, the mixed discriminant $D\left(A_{1} \cdots A_{n}\right)$ is nonnegative.

From now on, we assume that the matrices $A_{1} \cdots A_{n}$ are positive semidefinite.

Mixed discriminants generalize permanents: If the matrices $A_{1} \cdots A_{n}$ are diagonal, namely $A_{j}=\operatorname{diag}\left(b_{1 j} \cdots b_{n j}\right)$, for $j=1, \ldots, n$, let $B=\left(b_{i j}\right)$. Then $\operatorname{per}(B)=$ $D\left(A_{1} \cdots A_{n}\right)$. It follows that computing the mixed discriminant of $n$ positive semidefinite matrices is \# $P$-hard, since it is at least as hard as computing the permanent of a nonnegative matrix.

A positive definite $n \times n$ matrix $A$ defines an ellipsoid in $\mathbf{R}^{n}$, by setting $\mathcal{E}_{A}=\{x \in$ $\left.\mathbf{R}^{n}:\langle x, A x\rangle \leq 1\right\}$. The following relation between the mixed discriminant of positive definite matrices and the mixed volume of ellipsoids was established in [6]:

$$
3^{-(n+1) / 2} v_{n} D^{1 / 2}\left(A_{1}^{-1} \cdots A_{n}^{-1}\right) \leq V\left(\mathcal{E}_{A_{1}} \cdots \mathcal{E}_{A_{n}}\right) \leq v_{n} D^{1 / 2}\left(A_{1}^{-1} \cdots A_{n}^{-1}\right) .
$$

Here $v_{n}$ is the volume of the unit ball in $\mathbf{R}^{n}$.

Recall, that for any convex body $K$ in $\mathbf{R}^{n}$ there exists [19] an ellipsoid $\mathcal{E}_{K}$, such that (after translating its center to the origin) $\mathcal{E}_{K} \subseteq K \subseteq n \mathcal{E}_{K}$. For a well-presented convex body $K$ an ellipsoid $\mathcal{E}_{K}^{\prime}$ such that $\mathcal{E}_{K}^{\prime} \subseteq K \subseteq n \sqrt{n+1} \mathcal{E}_{K}^{\prime}$ can be constructed efficiently [15].

Barvinok [6], [7] gives an efficient polynomial-time probabilistic algorithm for approximating the mixed discriminant of $n$ positive semidefinite matrices with a $2^{O(n)}$ factor. Using the relations between the permanent, the mixed discriminant and the mixed

\footnotetext{
${ }^{2}$ Recall that a convex body in $\mathbf{R}^{n}$ is proper if its interior is not empty.

${ }^{3}$ Here and henceforth the sign $\preceq$ denotes the partial ordering induced by the cone of positive semidefinite matrices, namely $A \preceq B$ iff $B-A$ is positive semidefinite.
} 
volume of ellipsoids (and the fact that every well-presented convex body can be efficiently approximated by an ellipsoid), he obtains approximation results for the permanent and the mixed volume.

Apart from their ties to permanents and mixed volumes, mixed discriminants also have independent applications to computationally hard problems of combinatorial counting, such as counting the "coloured spanning trees" [7].

\subsection{Our Results}

We achieve $n^{n} / n ! \approx e^{-n}$-factor polynomial-time approximation of the mixed discriminant deterministically.

Theorem 1.1. There is a function $f$ such that

$$
D\left(A_{1} \cdots A_{n}\right) \leq f\left(A_{1} \cdots A_{n}\right) \leq \frac{n^{n}}{n !} \cdot D\left(A_{1} \cdots A_{n}\right)
$$

holds on every $n$-tuple of positive semidefinite $n \times n$ matrices $A_{i}$. The function $f$ is computable in time polynomial in $n$ and $\log v$, where $v$ is the maximal binary representation length of the entries of $A_{1} \cdots A_{n}$.

Similarly to [6], we obtain mixed volume approximation results, using Theorem 1.1, (2) and the efficient approximation of convex bodies by ellipsoids.

Theorem 1.2. There is a function $g$ such that

$$
V\left(K_{1} \cdots K_{n}\right) \leq g\left(K_{1} \cdots K_{n}\right) \leq n^{O(n)} \cdot V\left(K_{1} \cdots K_{n}\right)
$$

holds on every $n$-tuple of proper well-presented convex bodies $K_{i}$ in $\mathbf{R}^{n}$. The function $g$ is computable in time polynomial in $n$ and the presentation size of the bodies.

Our approach to this problem follows the approach of [21]. In short, we reduce the question of approximating mixed discriminants of $n$-tuples to that of approximating mixed discriminants on a smaller class of doubly stochastic $n$-tuples. The reduction technique is $n$-tuple scaling. We then use bounds on the mixed discriminant of doubly stochastic $n$-tuples to obtain the desired approximation. We remark that the tight upper bound of 1 is trivial, while the tight lower bound of $n ! / n^{n}$ is a generalization of the Egorychev-Falikman theorem. This bound was very recently proved by the first author [16].

Definition 1.3. Let $\mathbf{A}=\left(A_{1} \cdots A_{n}\right)$ and $\mathbf{B}=\left(B_{1} \cdots B_{n}\right)$ be two $n$-tuples of $n \times n$ matrices. The tuple $\mathbf{B}$ is a scaling of $\mathbf{A}$ if there is a vector $x \in \mathbf{R}^{n}$ and two $n \times n$ matrices $T_{1}, T_{2}$, such that $B_{i}=x_{i} T_{1} A_{i} T_{2}$, for all $i=1, \ldots, n$.

An important property of scaling is that we know how it changes the mixed discriminant. 


\section{Lemma 1.4.}

$$
D(\mathbf{B})=\prod_{i=1}^{n} x_{i} \cdot \operatorname{det} T_{1} \cdot \operatorname{det} T_{2} \cdot D(\mathbf{A})
$$

Proof. The claim easily follows from the definition of the mixed discriminant and the multiplicative property of the determinant: $\operatorname{det}(A B)=\operatorname{det}(A) \operatorname{det}(B)$.

Definition 1.5 [3]. An $n$-tuple $\mathbf{A}=\left(A_{1} \cdots A_{n}\right)$ of positive semidefinite matrices is doubly stochastic if

$$
\forall i, \quad \operatorname{Tr}\left(A_{i}\right)=1 \quad \text { and } \quad \sum_{i} A_{i}=I
$$

$I$ is the identity matrix here and from now on.

Definition 1.6. Let $\mathbf{A}=\left(A_{1} \cdots A_{n}\right)$ be an $n$-tuple of $n \times n$ positive semidefinite matrices. A positive vector $x \in \mathbf{R}^{n}$ and a positive definite $n \times n$ matrix $S$ are scaling factors of $\mathbf{A}$ if the $n$-tuple $\mathbf{B}=\left(B_{1} \cdots B_{n}\right)$ given by $B_{i}=x_{i} S^{1 / 2} A_{i} S^{1 / 2}$ is doubly stochastic. $^{4}$

So far we have given a very "small scale" overview of things. In the next subsection we go into details.

\subsection{An Overview of the Mixed Discriminant Approximation Algorithm}

- We define a notion of a fully indecomposable tuple,

Definition 1.7. An $n$-tuple $\mathbf{A}=\left(A_{1}, \ldots, A_{n}\right)$ of positive semidefinite $n \times n$ matrices is fully indecomposable if for all $S \subseteq\{1, \ldots, n\}, 0<|S|<n$, $\operatorname{Rank}\left(\sum_{i \in S} A_{i}\right)>|S|$.

and show a reduction of the problem to the case of fully indecomposable tuples. This is done in Section 2.

- We show that the problem of the existence and computation of scaling factors for an indecomposable tuple can be translated to determining whether an explicitly given convex function obtains a minimum over a specific convex set, and to finding this minimum. We deduce the existence (and uniqueness) of scaling factors for an indecomposable tuple. This is done in Section 3.

- We give an approximate solution of this convex optimization problem using the Ellipsoid method.

This, together with Lemma 1.4, reduces the problem to the case of doubly stochastic tuples. This is done in Section 4.

\footnotetext{
${ }^{4}$ Here $S^{1 / 2}$ is the unique positive semidefinite matrix whose square is $S$.
} 
Remark 1.8. Matrix scaling and, we believe, $n$-tuple scaling as well, are important problems, even without their ties to permanents and mixed discriminants. Matrix scaling problems were solved via a convex programming approach in [20] and, in a more general setting, in [22].

A principal step in establishing complexity bounds for a convex programming approach is to get an a priori bound on a solution, i.e., an upper bound for the variation of the convex function on an ellipsoid which contains a solution, and this was the main technical part of both [20] and [22]. It is interesting that the notion of a mixed discriminant enters naturally in obtaining the corresponding upper bound in our case (Lemma 4.1).

- We conclude by applying the bounds on the mixed discriminant of doubly stochastic tuples.

The following four theorems correspond to the four clauses above.

Theorem 1.9. Let $\mathbf{A}=\left(A_{1}, \ldots, A_{n}\right)$ be an $n$-tuple of positive semidefinite matrices with a positive mixed discriminant. Then there is an integer $1 \leq k \leq n$, a positive constant $c$ and fully indecomposable tuples $\mathbf{B}_{1} \cdots \mathbf{B}_{k}$ of positive semidefinite matrices, such that

$$
D(\mathbf{A})=c \cdot \prod_{s=1}^{k} D\left(\mathbf{B}_{s}\right) .
$$

The tuples $\mathbf{B}_{1} \cdots \mathbf{B}_{k}$ and the constant $c$ can be found in polynomial time.

Theorem 1.10. Let $\mathbf{A}=\left(A_{1}, \ldots, A_{n}\right)$ be a fully indecomposable $n$-tuple of positive semidefinite matrices. Then:

1. There exist scaling factors $x$ and $S$ such that $\mathbf{B}=\left(x_{1} S^{1 / 2} A_{1} S^{1 / 2}, \ldots\right.$, $\left.x_{n} S^{1 / 2} A_{n} S^{1 / 2}\right)$ is doubly stochastic.

2. Let there be two pairs of scaling factors $(x, S)$ and $\left(x^{\prime}, S^{\prime}\right)$ for $\mathbf{A}$, and assume a normalization $\prod_{i=1}^{n} x_{i}=\prod_{i=1}^{n} x_{i}^{\prime}=1$. Then $x_{i}=x_{i}^{\prime}$ for all $1 \leq i \leq n$ and $S^{\prime}=S$.

Definition 1.11. Let $\mathbf{A}=\left(A_{1} \cdots A_{n}\right)$ be an $n$-tuple of $n \times n$ positive semidefinite matrices. A positive vector $x \in \mathbf{R}^{n}$ and a positive definite $n \times n$ matrix $S$ are $\varepsilon$-scaling factors for $\mathbf{A}$, if the $n$-tuple $\mathbf{B}=\left(B_{1} \cdots B_{n}\right)$, given by $B_{i}=x_{i} S^{1 / 2} A_{i} S^{1 / 2}$, is $\varepsilon$-doubly stochastic, namely

$$
\sum_{i=1}^{n}\left(\operatorname{Tr}\left(B_{i}\right)-1\right)^{2} \leq \varepsilon^{2}
$$

and

$$
\sum_{i=1}^{n} B_{i}=I
$$


Theorem 1.12. Let $\mathbf{A}=\left(A_{1}, \ldots, A_{n}\right)$ be a fully indecomposable $n$-tuple of positive semidefinite $n \times n$ matrices. Let $\varepsilon>0$ be a required scaling accuracy. Then $\varepsilon$-scaling factors $x_{1}^{\prime} \cdots x_{n}^{\prime}$ and $S^{\prime}$ for $\mathbf{A}$ can be found in

$$
O\left(n^{5} \log \left(\frac{n v}{\varepsilon}\right)\right)
$$

arithmetic operations. Here $v$ is the maximal binary representation length of the entries in $A_{1} \cdots A_{n}$. Moreover, if $x_{1} \cdots x_{n}$ and $S$ are the proper scaling factors for $\mathbf{A}$, then

$$
\operatorname{det} S \cdot \prod_{i=1}^{n} x_{i} \leq \operatorname{det} S^{\prime} \cdot \prod_{i=1}^{n} x_{i}^{\prime} \leq\left(1+\varepsilon^{2}\right) \operatorname{det} S \cdot \prod_{i=1}^{n} x_{i} .
$$

Theorem 1.13 [16]. Let $\mathbf{A}=\left(A_{1}, \ldots A_{n}\right)$ be a doubly stochastic $n$-tuple of positive semidefinite $n \times n$ matrices. Then

$$
\frac{n !}{n^{n}} \leq D(\mathbf{A}) \leq 1
$$

Theorem 1.1 follows by combining Theorems 1.9, 1.10, 1.12 and 1.13.

\subsection{Corollaries}

The following result is proved in Section 5. It is an easy by-product of Theorems 1.9, $1.10,1.12$ and 1.13 .

Theorem 1.14. Let $A_{1}, \ldots, A_{n}$ be $n \times n$ positive semidefinite matrices and let $r_{1} \geq$ $r_{2} \geq \cdots \geq r_{n}>0$ be positive real numbers, such that for any $k$-set $\alpha \subseteq\{1 \cdots n\}$ the matrix $A_{\alpha}=\sum_{i \in \alpha} A_{i}$ has at least $k$ eigenvalues greater than or equal to $r_{k}$. Then

$$
D\left(A_{1} \cdots A_{n}\right) \geq \frac{n !}{n^{n}} \cdot \prod_{k=1}^{n} r_{k}
$$

We use this theorem to prove two corollaries of a combinatorial/geometric flavor.

The first proposition is a straightforward attempt to obtain a similar statement for mixed volumes. A $k$-dimensional section of a set in $\mathbf{R}^{n}$ is its intersection with a $k$ dimensional affine subspace.

Proposition 1.15. Let $K_{1}, \ldots, K_{n}$ be proper convex bodies in $\mathbf{R}^{n}$ and let $s>0$ be a real number, such that for any $k$-set $\alpha \subseteq\{1 \cdots n\}$ the body $A_{\alpha}=\sum_{i \in \alpha} A_{i}$ has a $k$-dimensional section containing a translation of $s \cdot B_{k}$. Here $B_{k}$ is a $k$-dimensional Euclidean unit ball. Then

$$
M\left(K_{1} \cdots K_{n}\right) \geq\left(\Omega\left(s n^{-5 / 2}\right)\right)^{n} .
$$

The second claim generalizes a theorem of Rado, which states that $n$ families of vectors $U_{1} \cdots U_{n}$ in $\mathbf{R}^{n}$ have a linearly independent transversal (namely a choice of 
vectors $u_{1} \in U_{1}, \ldots, u_{n} \in U_{n}$ such that $u_{1} \cdots u_{n}$ are linearly independent) iff for any $\alpha \subseteq\{1 \cdots n\}$ the family $\bigcup_{i \in \alpha} U_{i}$ contains $|\alpha|$ independent vectors.

Theorem 1.16. Let $U_{1} \cdots U_{n}$ be $n$ families of vectors in $\mathbf{R}^{n}$ and let $\varepsilon>0$ be a real number, such that for any $k$-set $\alpha \subseteq\{1 \cdots n\}$ the family $U_{\alpha}=\bigcup_{i \in \alpha} U_{i}$ contains $k$ vectors $v_{1} \cdots v_{k}$ with

$$
\operatorname{Vol}_{k}\left(\left[v_{1} \cdots v_{k}\right]\right) \geq \varepsilon^{k} .
$$

Here $\left[v_{1} \cdots v_{k}\right]$ is the $k$-dimensional box spanned by $v_{1} \cdots v_{k}$, and $\mathrm{Vol}_{k}$ denotes the $k$-dimensional volume. Let the maximal length of a vector in $\bigcup_{i=1}^{n} U_{i}$ be bounded by $\ell$. Then there is a choice of vectors $u_{1} \in U_{1}, \ldots, u_{n} \in U_{n}$ such that

$$
\operatorname{Vol}_{n}\left(\left[v_{1} \cdots v_{n}\right]\right) \geq\left(\frac{1}{e^{1 / 2} n}\right)^{n / 2} \cdot 2^{-n^{2} / 2} \cdot\left(\frac{\varepsilon}{\ell}\right)^{n(n+1) / 2} \cdot \ell^{n} .
$$

\section{Reduction to the Fully Indecomposable Case}

We start by quoting two properties of the mixed discriminant. First, another representation [3]:

$$
D\left(A_{1}, \ldots, A_{n}\right)=\sum_{\sigma \in S_{n}} \operatorname{det}\left(A_{\sigma}\right),
$$

where $A_{\sigma}$ is the $n \times n$ matrix whose $i$ th column is the $i$ th column of $A_{\sigma(i)}$.

Next, a positivity criterion.

Theorem 2.1 [24]. Let $\mathbf{A}=\left(A_{1}, \ldots, A_{n}\right)$ be an $n$-tuple of positive semidefinite matrices. Then the following two conditions are equivalent:

1. $D(\mathbf{A})>0$.

2. For all $\alpha \subseteq\{1, \ldots, n\}, 0<|\alpha| \leq n$, $\operatorname{Rank}\left(\sum_{i \in \alpha} A_{i}\right) \geq|\alpha|$.

Now we proceed with the proof of the main result of this section, Theorem 1.9.

First, we point out that one can check in polynomial time whether the mixed discriminant of a given $n$-tuple of positive semidefinite matrices vanishes. (Recall that it is always nonnegative.)

Lemma 2.2. Let $\mathbf{A}$ be an $n$-tuple of positive semidefinite matrices. There is a polynomial-time algorithm which decides whether $D(\mathbf{A})=0$ or $D(\mathbf{A})>0$.

Proof. We follow the argument of [9, Theorem 8] that solves a similar problem of determining whether a mixed volume of $n$ convex well-presented bodies is zero.

Let $E_{i}=\left\{a_{i}^{1}, \ldots, a_{i}^{n}\right\}$ be the set of columns of $A_{i}$. Recall that for positive semidefinite matrices $A, B$ it holds that $\operatorname{Im}(A+B)=\operatorname{Im}(A)+\operatorname{Im}(B)$, and, therefore, for any $\alpha \subseteq\{1 \cdots n\}$ it holds that $\operatorname{Im}\left(\sum_{i \in \alpha} A_{i}\right)=\operatorname{Span}\left(\bigcup_{i \in \alpha} E_{i}\right)$. Theorem 2.1 implies then that $D(\mathbf{A})>0$ iff for any $\alpha \subseteq\{1 \cdots n\}$ the set $\bigcup_{i \in \alpha} E_{i}$ has at least $|\alpha|$ independent 
vectors. By a theorem of Rado, this is true if and only if $E_{1} \cdots E_{n}$ have a linearly independent transversal.

Consider two matroids on the ground set $E=\bigcup_{i=1}^{n} E_{i}$. The first is the linear matroid in which the independent sets are the linear independent subsets of $E$. The second is the transversal matroid, the bases of which are the transversals of the family $\left\{E_{1}, \ldots, E_{n}\right\}$. To determine whether $E_{1} \cdots E_{n}$ have a linearly independent transversal, we have to solve a 2-matroid intersection problem. Since the complexity of this problem is known [10] to be polynomial, we are done.

Proof of Theorem 1.9. If $\mathbf{A}$ is a decomposable $n$-tuple with a positive mixed discriminant, then there exists $\alpha \subset[n]$, with $\operatorname{Rank}\left(\sum_{i \in \alpha} A_{i}\right)=|\alpha|$. Our first step is to find a minimal nonempty set $\alpha$ with this property, or to decide that $\mathbf{A}$ is indecomposable, in which case we are done. For this purpose we consider $n(n-1)$ auxiliary $n$-tuples $\mathbf{A}^{i j}$, where $\mathbf{A}^{i j}$ is obtained from $\mathbf{A}$ by substituting $A_{i}$ instead of $A_{j}$. Let $D_{i j}=D\left(\mathbf{A}^{i j}\right)$. We define an $n \times n$ matrix $Z$ by $Z_{i j}=0$ if $D_{i j}=0$, and $Z_{i j}=1$ otherwise. By Lemma 2.2, the matrix $Z$ is constructible in polynomial time. The next lemma explains how this matrix highlights the sets we are looking for.

Lemma 2.3. Let $\emptyset \neq \alpha \subseteq[n]$. The following two statements are equivalent:

1. $\operatorname{Rank}\left(\sum_{i \in \alpha} A_{i}\right)=|\alpha|$, and $\alpha$ contains no proper nonempty subsets with this property.

2. $Z_{i j}=1$ for all $i, j \in \alpha$ and $Z_{i j}=0$ for all $i \in \alpha$ and $j \notin \alpha$.

The proof of this lemma is essentially the same as that of Lemma 3.3. We refer to the forthcoming proof of that lemma.

Consider $Z$ as an adjacency matrix of a directed graph $G=([n], E)$, where $e=i \rightarrow j$ belongs to $E$ iff $Z_{i j}=1$. For $i \in[n]$, let $W_{i}$ be the set of points in $G$ which can be reached from $i$. Lemma 2.3 implies that $\alpha$ is a minimal set with the property $\operatorname{Rank}\left(\sum_{i \in \alpha} A_{i}\right)=|\alpha|$ iff, for any $i \in \alpha, W_{i}=\alpha$ holds, and, moreover, $\alpha$ is a clique of $G$. We compute the sets $W_{i}$ for all $i \in[n]$ and check whether $W_{i}$ is a clique. If it holds for some $i \in[n]$ and $W_{i} \subset G$, we set $\alpha=W_{i}$, otherwise $\mathbf{A}$ is indecomposable and we are done.

Let $S=\sum_{i \in \alpha} A_{i}$ and let $X=\operatorname{Im}(S)$. Let $v_{1}, \ldots, v_{c}$ be an orthogonal basis of $X$, and let $v_{1}, \ldots, v_{n}$ be its completion to an orthogonal basis of $\mathbf{R}^{n}$. Let $U$ be the $n \times c$ matrix with columns $v_{1}, \ldots, v_{c}$ and let $U^{\perp}$ be the $n \times(n-c)$ matrix with columns $v_{c+1}, \ldots, v_{n}$. We set $B_{i}=U^{t} A_{i} U$ for $i \in \alpha$, and $\tilde{A}_{j}=\left(U^{\perp}\right)^{t} A_{j} U^{\perp}$ for $j \notin \alpha$. Then:

Lemma 2.4.

$$
D\left(A_{1}, \ldots, A_{n}\right)=\frac{1}{\prod_{i=1}^{n}\left\langle v_{i}, v_{i}\right\rangle} D\left(\left(B_{i}\right)_{i \in \alpha}\right) \cdot D\left(\left(\tilde{A}_{j}\right)_{j \notin \alpha}\right) .
$$

Proof. Let $V$ be the matrix with columns $v_{1} \cdots v_{n}$. Clearly, $\operatorname{det}(V)=\operatorname{det}\left(V^{t}\right)=$ $\prod_{i=1}^{n} \sqrt{\left\langle v_{i}, v_{i}\right\rangle}$. By Lemma 1.4, we have $D\left(A_{1} \cdots A_{n}\right)=\left(1 / \prod_{i=1}^{n}\left\langle v_{i}, v_{i}\right\rangle\right) D\left(V^{t} A_{1} V \cdots\right.$ $V^{t} A_{n} V$ ). Observe that, for $i \in \alpha$, the matrix $V^{t} A_{i} V$ is zero everywhere, but on a $c \times c$ upper left submatrix, and this submatrix is $B_{i}$. Observe also that, for $j \notin \alpha$, the lower right $(n-c) \times(n-c)$ submatrix of $V^{t} A_{j} V$ is precisely $\tilde{A}_{j}$. Using representation (10) 
of the mixed discriminant, it is not hard to see that in such a case the mixed discriminant decomposes: $D\left(V^{t} A_{1} V \cdots V^{t} A_{n} V\right)=D\left(\left(B_{i}\right)_{i \in \alpha}\right) \cdot D\left(\left(\tilde{A}_{j}\right)_{j \notin \alpha}\right)$.

Now we proceed inductively (on dimension) with the $(n-c)$-tuple $\left(\tilde{A}_{j}\right)_{j \notin \alpha}$. We point out that the minimality of $\alpha$ implies the indecomposability of the tuple $\left(B_{i}\right)_{i \in \alpha}$.

It remains to estimate the cost that we pay for decomposing the $n$-tuple $\mathbf{A}$. We perform $O(n)$ steps. In each of these steps the heaviest part by far is constructing the 0-1 matrix $Z$, which entails checking $\Omega\left(n^{2}\right)$ mixed discriminants for being zero. The total cost is, therefore, polynomial, and we are done.

The only thing which has yet to be pointed out is that the representation length $v_{i}$ of the components $\mathbf{B}_{i}$ is not much greater than the representation length $v$ of $\mathbf{A}$. Indeed, in the course of the decomposition procedure the representation length increases, essentially, only in construction of orthogonal bases. A moment's reflection gives that the multiplicative factor of the increase is at most exponential ${ }^{5}$ in $n$.

Remark 2.5. We call an $n$-tuple $\mathbf{A}=\left(A_{1} \cdots A_{n}\right)$ of positive semidefinite matrices scalable if it has a doubly stochastic scaling. The preceding proof provides a nice characterization of scalability: the $n$-tuple $\mathbf{A}=\left(A_{1} \cdots A_{n}\right)$ of positive semidefinite matrices is scalable if and only if the matrix $Z_{\mathbf{A}}=\left(\operatorname{sign}\left(D\left(\mathbf{A}^{i j}\right)\right)\right)$ is symmetric.

Indeed, it is not hard to see that (in the notation of the proof) the matrix $Z_{\mathbf{A}}$ is symmetric if and only if there is a $k$-partition $\{1 \cdots n\}=C_{1} \cup C_{2} \cup \cdots \cup C_{k}$ of the interval, such that the subspaces $X_{s}:=\operatorname{Im}\left(\sum_{i \in C_{s}} A_{i}\right), s=1 \cdots k$, decompose $\mathbf{R}^{n}$ into a direct sum of orthogonal subspaces. Moreover, for all $1 \leq s \leq k$, the tuple $\mathbf{B}_{s}$ is a projection of $\left(A_{i}\right)_{i \in C_{s}}$ onto $X_{s}$.

It follows that scaling factors for A can be obtained as an appropriate "concatenation" of the scaling factors for $\mathbf{B}_{1} \cdots \mathbf{B}_{k}$.

On the other hand, assume $\mathbf{A}$ has a doubly stochastic scaling, and let $\mathbf{B}$ be a corresponding doubly stochastic tuple. Then clearly $Z_{\mathbf{A}}=Z_{\mathbf{B}}$. It is not hard to check that for a doubly stochastic tuple the matrix $Z$ is symmetric, and the claim follows.

\section{A Convex Minimization Problem. Existence and Uniqueness of Scaling Factors}

Given an $n$-tuple of positive semidefinite matrices, we now define a convex function whose minima correspond to the scaling factors of the tuple.

Definition 3.1. Let $\mathbf{A}=\left(A_{1} \cdots A_{n}\right)$ be an $n$-tuple of positive semidefinite matrices. We define

$$
f\left(\xi_{1}, \ldots, \xi_{n}\right)=f_{\mathbf{A}}\left(\xi_{1}, \ldots, \xi_{n}\right)=\log \operatorname{det}\left(e^{\xi_{1}} A_{1}+\cdots+e^{\xi_{n}} A_{n}\right)
$$

\footnotetext{
${ }^{5}$ Note that, since our scaling algorithm runs in time logarithmic in the representation length, this increase is tolerable.
} 
Lemma 3.2. The function $f$ is a convex function on $\mathbf{R}^{n}$, and if $\mathbf{A}$ is fully indecomposable, then $f$ is strictly convex ${ }^{6}$ on the hyperplane $H=\left\{\xi=\left(\xi_{1}, \ldots, \xi_{n}\right) \in \mathbf{R}^{n} \mid \sum \xi_{i}=0\right\}$.

Before proving this lemma we introduce a lemma and a definition which attempt to "quantify" the indecomposability of $\mathbf{A}$. Consider once again the $n(n-1) n$-tuples $\mathbf{A}^{i j}$, where $\mathbf{A}^{i j}$ is obtained from $\mathbf{A}$ by substituting $A_{i}$ instead of $A_{j}$. Let $D_{i j}=D\left(\mathbf{A}^{i j}\right)$.

Lemma 3.3. A is indecomposable if and only if $D_{i j}>0$ for all $i, j$.

Proof. Assume first that $\mathbf{A}=\left(A_{1}, \ldots, A_{n}\right)$ is indecomposable. We claim that for any $1 \leq i \neq j \leq n$ the tuple $\mathbf{A}^{i j}=\left(A_{1}^{\prime}, \ldots, A_{n}^{\prime}\right)$ satisfies property 2 of Theorem 2.1, and therefore its mixed determinant is positive. Indeed, let $R \subseteq\{1 \cdots n\}$. Then $\operatorname{Rank}\left(\sum_{k \in R} A_{k}^{\prime}\right) \geq \operatorname{Rank}\left(\sum_{k \in R \backslash\{j\}} A_{k}\right) \geq|R|$, by the indecomposability of $\mathbf{A}$.

In the other direction, let $\mathbf{A}=\left(A_{1}, \ldots, A_{n}\right)$ be a decomposable tuple, namely for some subset $R \subset\{1 \cdots n\}$, the inequality $\operatorname{Rank}\left(\sum_{k \in R} A_{k}\right) \leq|R|$ holds. Let $i, j$ be a pair of indices such that $i \in R$ and $j \notin R$, and consider the tuple $\mathbf{A}^{i j}=\left(A_{1}^{\prime}, \ldots, A_{n}^{\prime}\right)$. We claim that $D\left(\mathbf{A}^{i j}\right)=0$. Indeed,

$$
\operatorname{Rank}\left(\sum_{k \in R \cup\{j\}} A_{k}^{\prime}\right)=\operatorname{Rank}\left(\sum_{k \in R} A_{k}\right)<|R|+1=|R \cup\{j\}|,
$$

which, by Theorem 2.1, implies $D\left(\mathbf{A}^{i j}\right)=0$.

Lemma 3.3 suggests the following quantitative measure of indecomposability:

Definition 3.4. Set

$$
M=M_{\mathbf{A}}=\min _{i \neq j} D_{i j}
$$

Proof of Lemma 3.2. By definition, the coefficient of $x_{1} x_{2} \cdots x_{n}$ in the polynomial $\operatorname{det}\left(x_{1} A_{1}+\cdots+x_{n} A_{n}\right)$ is the mixed discriminant $D\left(A_{1} \cdots A_{n}\right)$. It turns out [3] that all the coefficients of this polynomial can be expressed through mixed discriminants. Let $r_{1}, \ldots, r_{n}$ be nonnegative integers adding to $n$. Then the coefficient of $x_{1}^{r_{1}} \cdots x_{n}^{r_{n}}$ is equal to

$$
t_{r}=\frac{1}{r_{1} ! \cdots r_{n} !} D(\underbrace{A_{1} \cdots A_{1}}_{r_{1}} \cdots \underbrace{A_{n} \cdots A_{n}}_{r_{n}}) .
$$

We denote the set of $n$-tuples $r=r_{1}, \ldots, r_{n}$ of nonnegative integers summing to $n$ by $P_{n}$. In this notation

$$
f\left(\xi_{1}, \ldots, \xi_{n}\right)=\log \operatorname{det}\left(e^{\xi_{1}} A_{1}+\cdots+e^{\xi_{n}} A_{n}\right)=\log \sum_{r \in P_{n}} t_{r} e^{\langle\xi, r\rangle}
$$

${ }^{6}$ Namely, for all $\xi, \xi^{\prime}$, and $0<\lambda<1$, it holds that $f\left(\lambda \xi+(1-\lambda) \xi^{\prime}\right)<\lambda f(\xi)+(1-\lambda) f\left(\xi^{\prime}\right)$. 
where the coefficients $t_{r}$ are nonnegative, since they are given by a mixed discriminant of positive semidefinite matrices multiplied by a multinomial coefficient, and $\langle$,$\rangle is the$ usual inner product in $\mathbf{R}^{n}$. It is well known [2] that the right-hand side represents a convex function of $\xi$. Nonetheless, we provide another proof of this fact, which will also imply that $f$ is strictly convex on $H$ if the tuple $A_{1} \cdots A_{n}$ is indecomposable.

Let $g(\xi)=\operatorname{det}\left(e^{\xi_{1}} A_{1}+\cdots+e^{\xi_{n}} A_{n}\right)$, namely $f=\log g$. To show that $f$ is convex we have to show that its Hessian $\nabla^{2} f$ is positive semidefinite. Clearly, $\nabla^{2} f=$ $\left(1 / g^{2}\right)\left(g\left(\nabla^{2} g\right)-(\nabla g)(\nabla g)^{t}\right)$. Therefore, we have to show $g \nabla^{2} g \succeq(\nabla g)(\nabla g)^{t}$. For two vectors $v, w \in \mathbf{R}^{n}$, let $v \otimes w$ denote the matrix $v w^{t}$. Note that $v \otimes v$ is positive semidefinite. Observe that for any $v \in \mathbf{R}^{n}$ it holds that $\nabla e^{\langle\xi, v\rangle}=e^{\langle\xi, v\rangle} \cdot v$ and $\nabla^{2} e^{\langle\xi, v\rangle}=$ $e^{\langle\xi, v\rangle} \cdot v \otimes v$, and therefore

$$
\begin{aligned}
g\left(\nabla^{2} g\right)-(\nabla g)(\nabla g)^{t} & =\sum_{r \in P_{n}} t_{r} e^{\langle\xi, r\rangle} \cdot \sum_{s \in P_{n}} t_{s} e^{\langle\xi, s\rangle} s \otimes s-\sum_{r, s \in P_{n}} t_{r} t_{s} e^{\langle\xi, r+s\rangle} r \otimes s \\
& =\frac{1}{2} \sum_{r, s \in P_{n}} t_{r} t_{s} e^{\langle\xi, r+s\rangle}(r-s) \otimes(r-s) \succeq 0,
\end{aligned}
$$

implying the convexity of $f$.

Now, assume the tuple $A_{1}, \ldots, A_{n}$ to be indecomposable. Recall that $D_{i j}$ is the mixed discriminant of the $n$-tuple obtained from $\left(A_{1}, \ldots, A_{n}\right)$ by replacing $A_{j}$ with $A_{i}$, and that $M=\min _{i \neq j} D_{i j}$ is positive. In the notation of this lemma, $D_{i j}$ is just $2 t_{r_{i j}}$, where $r_{i j}$ is the vector with 1 in every coordinate but $i, j$, and with 2 in the $i$ th and 0 in the $j$ th coordinates. We now continue the computation from (12):

$$
\begin{aligned}
\nabla^{2} f & \succeq \frac{1}{2 g^{2}} \sum_{r, s \in P_{n}} t_{r} t_{s} e^{\langle\xi, r+s\rangle}(r-s) \otimes(r-s) \\
& \succeq \frac{1}{8 g^{2}} \sum_{i \neq j, k \neq l} D_{i j} D_{k l} e^{\left\langle\xi, r_{i j}+r_{k l}\right\rangle}\left(r_{i j}-r_{k l}\right) \otimes\left(r_{i j}-r_{k l}\right) \\
& \succeq \frac{c M^{2}}{8 g^{2}} \sum_{i \neq j \neq k \neq l}\left(r_{i j}-r_{k l}\right) \otimes\left(r_{i j}-r_{k l}\right),
\end{aligned}
$$

where the last summation is over distinct indices $i, j, k, l$. Here $c=c(\xi)=$ $\min _{i \neq j \neq k \neq l} e^{\left\langle\xi, r_{i j}+r_{k l}\right\rangle}$. Let $e_{i}$ be the $i$ th unit vector, and let $E_{i j}$ be the $n \times n$ matrix with 1 in the $(i, j)$ th coordinate and 0 everywhere else. Then

$$
\begin{aligned}
\left(r_{i j}-r_{k l}\right) \otimes\left(r_{i j}-r_{k l}\right)= & \left(e_{i}-e_{j}-e_{k}+e_{l}\right) \otimes\left(e_{i}-e_{j}-e_{k}+e_{l}\right) \\
= & E_{i i}+E_{j j}+E_{k k}+E_{l l} \\
& +2\left(E_{i l}+E_{j k}-E_{i j}-E_{i k}-E_{j l}-E_{k l}\right) .
\end{aligned}
$$

Let

$$
S=\sum_{i \neq j \neq k \neq l}\left[E_{i i}+E_{j j}+E_{k k}+E_{l l}+2\left(E_{i l}+E_{j k}-E_{i j}-E_{i k}-E_{j l}-E_{k l}\right)\right] .
$$

Finding $S$ might seem like a mess, but actually it is not. By symmetry considerations, the entries of $S$ have only two distinct values, on the main diagonal and every else, and 
moreover they sum to zero. Therefore, we only have to find the trace of $S$, and this is easily seen to be $(n)_{4}=n(n-1)(n-2)(n-3)$. Accordingly, $S_{i i}=(n-1)_{3}=$ $(n-1)(n-2)(n-3)$ and $S_{i j}=-(n-2)(n-3)$ for $i \neq j$. Note that $S$ is simply the orthogonal projection on the hyperplane $H=\left\{\xi=\left(\xi_{1}, \ldots, \xi_{n}\right) \in \mathbf{R}^{n} \mid \sum \xi_{i}=0\right\}$, multiplied by $(n-1)_{3}$.

Therefore, the projection of $\nabla^{2} f$ onto $H$ is greater than or equal to $\left(c M^{2} \cdot(n-\right.$ $\left.1)_{3} / 8 g^{2}\right) \cdot I_{n-1}$, where $I_{n-1}$ is an $(n-1) \times(n-1)$ identity matrix, implying $f$ is strictly convex on $H$.

Lemma 3.5. For any $\xi \in \mathbf{R}^{n}$, the gradient $(\nabla f)_{\xi}$ of $f$ at $\xi$ is $\left(\operatorname{Tr}\left(e^{\xi_{i}} S^{1 / 2} A_{i} S^{1 / 2}\right)\right)_{i=1}^{n}$, where $S=\left(\sum_{i=1}^{n} e^{\xi_{i}} A_{i}\right)^{-1}$.

Proof. Recall that the gradient $(\nabla \log \operatorname{det}(\cdot))_{B}$ of $f$ at a nonsingular matrix $B$ is just its inverse transposed $\left(B^{-1}\right)^{t}$. Therefore, taking $B=\left(\sum_{i=1}^{n} e^{\xi_{i}} A_{i}\right)$, and using the Chain Rule, we get that

$$
\left.\frac{\partial f}{\partial \xi_{i}}=\operatorname{Tr}\left((\nabla \log \operatorname{det}(\cdot))_{B}\right)\left(\frac{\partial B}{\partial \xi_{i}}\right)^{t}\right)=\operatorname{Tr}\left(S e^{\xi_{i}} A_{i}\right)=\operatorname{Tr}\left(e^{\xi_{i}} S^{1 / 2} A_{i} S^{1 / 2}\right) .
$$

Lemma 3.6. A point $\xi^{*}$ is a minimum of $f$ on $H$ if and only if the gradient $\nabla f$ at $\xi^{*}$ is a constant multiple of $(1 \cdots 1)$, the vector of all ones. In addition, $(\nabla f)_{\xi^{*}}=c \cdot(1 \cdots 1)$ if and only if $x_{i}=e^{\xi_{i}^{*}}, i=1, \ldots, n$, and $S=\left(\sum_{i=1}^{n} e^{\xi_{i}^{*}} A_{i}\right)^{-1}$, are the scaling factors for $\mathbf{A}$; namely the $n$-tuple $\left(x_{1} S^{1 / 2} A_{1} S^{1 / 2} \cdots x_{n} S^{1 / 2} A_{n} S^{1 / 2}\right)$ is doubly stochastic. In particular, $c=1$.

Proof. The first claim: Since $f$ is convex, $\xi^{*}$ is a point of minimum for $f$ on $H$ if and only if $(\nabla f)_{\xi^{*}}$ is a convex combination of the gradients at $\xi^{*}$ of the defining equations for $H$.

The second claim follows immediately from the first claim and Lemma 3.5.

The value of $M=\min _{i \neq j} D_{i j}$ plays a key part in the following lemma as well.

Lemma 3.7. Let $\xi \in H$ be such that $f(\xi) \leq f(0)$, then

$$
\|\xi\|_{2} \leq n^{1 / 2} \cdot \log \frac{2 \operatorname{det}\left(A_{1}+\cdots+A_{n}\right)}{M} .
$$

Proof. Let $\xi$ be a point in $H$ with $f(\xi) \leq f(0)=\log \operatorname{det}\left(A_{1}+\cdots+A_{n}\right)$. Then, in the notation of the proof of Lemma 3.2,

$$
\begin{aligned}
\operatorname{det}\left(A_{1}+\cdots+A_{n}\right) & \geq \operatorname{det}\left(e^{\xi_{1}} A_{1}+\cdots+e^{\xi_{n}} A_{n}\right) \\
& =\sum_{r \in P_{n}} t_{r} e^{\langle\xi, r\rangle} \geq \frac{1}{2} \sum_{i \neq j} D_{i j} e^{\left\langle\xi, r_{i j}\right\rangle} \geq \frac{1}{2} M \sum_{i \neq j} e^{\left\langle\xi, r_{i j}\right\rangle} \\
& \geq \frac{1}{2} M e^{\max _{i \neq j} \xi_{i}-\xi_{j}} \geq \frac{1}{2} M e^{\|\xi\|_{\infty} .}
\end{aligned}
$$

The last two inequalities use $\sum_{i} \xi_{i}=0$, which, in particular, implies $\left\langle\xi, r_{i j}\right\rangle=\xi_{i}-\xi_{j}$. 
Therefore,

$$
\|\xi\|_{2} \leq n^{1 / 2} \cdot\|\xi\|_{\infty} \leq n^{1 / 2} \cdot \log \frac{2 \operatorname{det}\left(A_{1}+\cdots+A_{n}\right)}{M} .
$$

Theorem 1.10 is a simple consequence of Lemma 3.6 and the following lemma which describes the behavior of minima of $f$ on $H$.

Lemma 3.8. The function $f$ attains a unique minimum on the hyperplane $H$.

Proof. By Lemma 3.2, $f$ is strictly convex on $H$. Therefore, the minimum $\xi^{*}$, if attained, is unique. On the other hand, by Lemma 3.7, the minimum of $f$ on $H$ is the minimum of $f$ on a ball with finite radius. Since this ball is compact, the minimum is attained.

Remark 3.9. We observe that, in the notation of Lemma 3.6,

$$
\begin{aligned}
f\left(\xi^{*}\right) & =\log \operatorname{det}\left(e^{\xi_{1}^{*}} A_{1}+\cdots+e^{\xi_{n}^{*}} A_{n}\right) \\
& =\log \operatorname{det}\left(S^{-1}\right)=\log \left(\frac{1}{\operatorname{det}(S) \cdot \prod_{i=1}^{n} x_{i}}\right)
\end{aligned}
$$

is the (log) product of the scaling factors of $\mathbf{A}$. Namely $D(\mathbf{A})=e^{f\left(\xi^{*}\right)} \cdot D(\mathbf{B})$, where $\mathbf{B}=\left(x_{1} S^{1 / 2} A_{1} S^{1 / 2} \cdots x_{n} S^{1 / 2} A_{n} S^{1 / 2}\right)$ is doubly stochastic.

\section{Finding the Minimum}

In the previous section we have seen that finding the point of minimum of the function $f=f_{\mathbf{A}}$ on the hyperplane $H$ is equivalent to computing the scaling factors of $\mathbf{A}$. This is interesting if we want to scale $\mathbf{A}$. We have also seen that finding the value of the minimum is equivalent to computing the product of the scaling factors of $\mathbf{A}$. This is sufficient for reduction of the mixed discriminant approximation problem to the doubly stochastic case. In this section we solve both questions. The solutions will be approximate, but with an arbitrary degree of precision.

Our main tool is the following property of the ellipsoid algorithm [23]: For a prescribed accuracy $\delta>0$, it finds a $\delta$-minimizer of a continuous convex function $f$ in a ball $B$, that is a point $x_{\delta} \in B$ with $f\left(x_{\delta}\right) \leq \min _{B} f+\delta$, in no more than

$$
O\left(n^{2} \ln \left(\frac{2 \delta+\operatorname{Var}_{B}(f)}{\delta}\right)\right), \quad \operatorname{Var}_{B}(f)=\max _{B} f-\min _{B} f,
$$

iterations. Each iteration requires a single computation of the value and of the gradient of $f$ at a given point, plus $O\left(n^{2}\right)$ elementary operations to run the algorithm itself. In our case, this is easily seen to cost at most $O\left(n^{3}\right)$ elementary operations.

Recall, that the radius $R$ of the ball $B$ is given by Lemma 3.7: $R \leq n^{1 / 2} \cdot \log \left(2 \operatorname{det}\left(A_{1}+\right.\right.$ $\left.\left.\cdots+A_{n}\right) / M\right)$. 


\section{Lemma 4.1.}

$$
\operatorname{Var}_{B}(f) \leq O\left(n^{5 / 2}(v+\log n)\right),
$$

where $v$ is the binary representation length of entries in $\mathbf{A}$.

Proof. We may, without loss of generality, assume that all the matrices $A_{i}$ in $\mathbf{A}$ have integer entries. Note that since the binary representation length of entries in $\mathbf{A}$ is $v$, the maximal size of an entry does not exceed $2^{v}$. By (10), since $M$ is greater than zero, it is at least one. On the other hand, by Hadamard's inequality, $\operatorname{det}\left(A_{1}+\cdots+A_{n}\right) \leq\left(n 2^{v}\right)^{n}=$ $n^{n} 2^{v n}$. Therefore, $R \leq n^{1 / 2} \cdot \log n^{n} 2^{v n}=n^{3 / 2}(v+\log n)$.

We conclude that $\max _{B} f \leq \log \left(e^{n R} \operatorname{det}\left(A_{1}+\cdots+A_{n}\right)\right) \leq O\left(n^{5 / 2}(v+\log n)\right)$.

On the other hand, the proof of Lemma 3.7 demonstrates that, for any $\xi \in H$, $f(\xi) \geq \log (M / 2) \geq-1$ holds. Therefore,

$$
\operatorname{Var}_{B}(f) \leq O\left(n^{5 / 2}(v+\log n)\right) .
$$

Proposition 4.2. Let $\mathbf{A}=\left(A_{1}, \ldots, A_{n}\right)$ be a fully indecomposable $n$-tuple of positive semidefinite matrices, and let $0<\varepsilon<1$. Let $\xi$ be an $\left(\varepsilon^{2} / 10\right)$-minimizer of $f$ on $H$. Then $x_{i}=e^{\xi_{i}}$, for $i=1, \ldots, n$, and $S=\left(\sum_{i=1}^{n} e^{\xi_{i}} A_{i}\right)^{-1}$ scale $\mathbf{A}$ to an $\varepsilon$-doubly stochastic tuple.

Proof. Let $\xi^{*}$ be the minimizer of $f$ on $H$. Let $\delta:=\varepsilon^{2} / 10$ and $A_{i}^{\prime}:=S^{1 / 2} e^{\xi_{i}} A_{i} S^{1 / 2}$. Since, by definition, $\sum_{i=1}^{n} A_{i}^{\prime}=I$, we only need to prove that $\sum_{i=1}^{n}\left(\operatorname{tr}\left(A_{i}^{\prime}\right)-1\right)^{2} \leq 10 \delta$.

We prove the proposition by a sequence of easy reductions to simpler cases. First we show that, in effect, we may assume $\mathbf{A}$ is doubly stochastic. We know that

$$
\begin{aligned}
\log \operatorname{det}\left(e^{\xi_{1}^{*}} A_{1}+\cdots+e^{\xi_{n}^{*}} A_{n}\right) & \leq \log \operatorname{det}\left(e^{\xi_{1}} A_{1}+\cdots+e^{\xi_{n}} A_{n}\right) \\
& \leq \log \operatorname{det}\left(e^{\xi_{1}^{*}} A_{1}+\cdots+e^{\xi_{n}^{*}} A_{n}\right)+\delta .
\end{aligned}
$$

Taking exponents and observing that, for a small $\delta, e^{\delta} \leq 1+2 \delta$ holds, we get

$\operatorname{det}\left(e^{\xi_{1}^{*}} A_{1}+\cdots+e^{\xi_{n}^{*}} A_{n}\right) \leq \operatorname{det}\left(e^{\xi_{1}} A_{1}+\cdots+e^{\xi_{n}} A_{n}\right) \leq \operatorname{det}\left(e^{\xi_{1}^{*}} A_{1}+\cdots+e^{\xi_{n}^{*}} A_{n}\right) \cdot(1+2 \delta)$.

Setting $S^{*}=\left(\sum_{i=1}^{n} e^{\xi^{*}} A_{i}\right)^{-1}, B_{i}=\left(S^{*}\right)^{1 / 2} e^{\xi_{i}^{*}} A_{i}\left(S^{*}\right)^{1 / 2}$ and $(\Delta \xi)_{i}=\xi_{i}-\xi_{i}^{*}$, we get

$$
1 \leq \operatorname{det}\left(e^{(\Delta \xi)_{1}} B_{1}+\cdots+e^{(\Delta \xi)_{n}} B_{n}\right) \leq 1+2 \delta .
$$

Observe that $\mathbf{B}=\left(B_{1}, \ldots, B_{n}\right)$ is a doubly stochastic tuple, by Lemma 3.6. For $i=$ $1, \ldots, n$, let $B_{i}^{\prime}:=\left(S^{\prime}\right)^{1 / 2} e^{(\Delta \xi)_{i}} B_{i}\left(S^{\prime}\right)^{1 / 2}$, where $S^{\prime}:=\left(\sum_{i=1}^{n} e^{(\Delta \xi)_{i}} B_{i}\right)^{-1}$. Then $B_{i}^{\prime}=$ $U^{t} A_{i}^{\prime} U$, where $U=S^{-1 / 2}\left(S^{*}\right)^{1 / 2}\left(S^{\prime}\right)^{1 / 2}$ is an orthogonal matrix. ${ }^{7}$ Clearly, $\operatorname{tr}\left(B_{i}^{\prime}\right)=$ $\operatorname{tr}\left(A_{i}^{\prime}\right)$, and $\sum_{i=1}^{n} B_{i}^{\prime}=\sum_{i=1}^{n} A_{i}^{\prime}=I$. Therefore, the claim of the proposition amounts to proving

$$
\sum_{i=1}^{n}\left(\operatorname{tr}\left(B_{i}^{\prime}\right)-1\right)^{2} \leq 10 \delta
$$

\footnotetext{
${ }^{7}$ We prove this: Observe that $S^{\prime}:=\left(\sum_{i=1}^{n} e^{(\Delta \xi)_{i}} B_{i}\right)^{-1}=\left(S^{*}\right)^{-1 / 2} S\left(S^{*}\right)^{-\frac{1}{2}}$. Therefore $U U^{t}=$ $S^{-1 / 2}\left(S^{*}\right)^{1 / 2} S^{\prime}\left(S^{*}\right)^{1 / 2} S^{-1 / 2}=S^{-1 / 2} S S^{-1 / 2}=I$.
} 
Next, we move from positive semidefinite doubly stochastic $n$-tuples to an easier case of doubly stochastic matrices. Let $W$ be an orthogonal matrix such that $W^{t} S^{\prime} W$ is diagonal, namely the columns $w_{1}, \ldots, w_{n}$ of $W$ are eigenvectors of $S^{\prime}$. Let $b_{j i}=\left\langle w_{j}, B_{i} w_{j}\right\rangle$. Then the matrix $B=\left(b_{i j}\right)$ is doubly stochastic, and (14) reduces to

$$
1 \leq \prod_{i=1}^{n}(B y)_{i} \leq 1+2 \delta,
$$

where $y \in \mathbf{R}^{n}$ is given by $y_{j}=e^{(\Delta \xi)_{j}}$, for $j=1, \ldots, n$. Note that

$$
\prod_{j=1}^{n} y_{j}=e^{\Sigma_{j} \xi_{j}-\Sigma_{j} \xi_{j}^{*}}=1
$$

Our claim amounts to showing, given $B$ is doubly stochastic, (15) and (16), that the matrix $C=\left(c_{i j}\right)=\left(b_{i j} y_{j} / \sum_{k=1}^{n} b_{i k} y_{k}\right)$ is $\varepsilon$-doubly stochastic. Clearly, $C$ is row-normalized. Setting $c_{j}=\sum_{i=1}^{n} c_{i j}$ to be the column sums of $C$, we have to show $\sum_{j=1}^{n}\left(c_{j}-1\right)^{2} \leq$ $\varepsilon^{2}$. Note that $\sum_{j=1}^{n} c_{j}=n$. We claim that, since $B$ is doubly stochastic, $\prod_{j=1}^{n} c_{j} \geq$ $\prod_{j=1}^{n} y_{j} / \prod_{i=1}^{n}(B y)_{i} \geq 1 /(1+2 \delta) \geq 1-2 \delta$. Only the first inequality has to be justified. Writing $C=\operatorname{diag}\left(1 /(B y)_{i}\right) \cdot B \cdot \operatorname{diag}\left(y_{j}\right)$, we obtain $\prod_{j=1}^{n} c_{j}=\prod_{j=1}^{n} y_{j} \cdot \prod_{i=1}^{n}(x B)_{i}$, where we have set $x_{i}=1 /(B y)_{i}$. It remains to use a well-known [4, p. 150] property of doubly stochastic matrices: for a nonnegative vector $x$ it holds that $\prod_{i=1}^{n}(x B)_{i} \geq$ $\prod_{i=1}^{n} x_{i}$.

Now we are in a familiar situation. Lemma 3.10 of [21] states that for nonnegative numbers $z_{1}, \ldots, z_{n}$ summing to $n$, and for a sufficiently small $\Delta\left(0 \leq \Delta \leq \frac{1}{10}\right.$ is enough), $\sum_{j=1}^{n}\left(z_{j}-1\right)^{2}=\Delta \Longrightarrow \prod_{j=1}^{n} z_{j} \leq 1-\Delta / 3$ holds. We deduce that in our case

$$
\sum_{j=1}^{n}\left(c_{j}-1\right)^{2} \leq 6 \delta<\varepsilon^{2}
$$

and we are done.

Theorem 1.12 follows from Lemma 4.1, Proposition 4.2 and the described properties of the ellipsoid method.

\section{Corollaries}

\subsection{Proof of Theorem 1.14}

Proof. By a perturbation argument, it suffices to prove the theorem for fully indecomposable $n$-tuples. So from now on we assume that $\mathbf{A}$ is indecomposable. We will show that the product of the scaling factors of $\mathbf{A}$ is at least $\prod_{i=1}^{n} r_{i}$. This, by Theorem 1.13, will complete the proof. 
By Remark 3.9, it suffices to show that for any $x_{1} \cdots x_{n}>0$ with $\prod_{i=1}^{n} x_{i}=1$ it holds that

$$
\operatorname{det}\left(x_{1} A_{1}+\cdots+x_{n} A_{n}\right) \geq \prod_{i=1}^{n} r_{i} .
$$

Indeed assume, without loss of generality, that the $x$ 's are ordered $x_{1} \geq x_{2} \geq \cdots \geq x_{n}$. For a symmetric matrix $A$, let $\lambda_{1}(A) \geq \lambda_{2}(A) \geq \cdots \geq \lambda_{n}(A)$ be the eigenvalues of $A$ in descending order. We will prove the claim in (17) showing that $\lambda_{k}\left(\sum_{i=1}^{n} x_{i} A_{i}\right) \geq r_{k} x_{k}$, for all $k=1, \ldots, n$. Recall, that if $A$ and $B$ are two symmetric matrices and $A-B$ is positive semidefinite, then $\lambda_{k}(A) \geq \lambda_{k}(B)$, for all $1 \leq k \leq n$. This follows, for instance from the Courant-Fischer theorem [14, p. 32]:

$$
\lambda_{k}(A)=\max _{\operatorname{dim}(U)=k} \min _{x \in U, x \neq 0} \frac{\langle x, A x\rangle}{\langle x, x\rangle} .
$$

Applying this fact twice gives

$$
\lambda_{k}\left(\sum_{i=1}^{n} x_{i} A_{i}\right) \geq \lambda_{k}\left(\sum_{i=1}^{k} x_{i} A_{i}\right) \geq \lambda_{k}\left(x_{k} \cdot \sum_{i=1}^{k} A_{i}\right) \geq r_{k} x_{k},
$$

proving (17) and the theorem.

\subsection{Proof of Proposition 1.15}

Proof. Let $K_{1}, \ldots, K_{n}$ be proper convex bodies in $\mathbf{R}^{n}$ and let $s>0$ be a real number, such that for any $k$-set $\alpha \subseteq\{1 \cdots n\}$ the body $\sum_{i \in \alpha} K_{i}$ has a $k$-dimensional section containing a translation of $s \cdot B_{k}$. We say, in brief, that $K_{1} \cdots K_{n}$ have an $s$-section property.

Let $\mathcal{E}_{1} \cdots \mathcal{E}_{n}$ be the John ellipsoids of these bodies, namely (after translating the center of $\mathcal{E}_{i}$ to the origin) we have $\mathcal{E}_{i} \subseteq K_{i} \subseteq n \mathcal{E}_{i}$, for $i=1, \ldots, n$. We may, and do, assume that the ellipsoids are, in fact, centered at the origin. Clearly, $\mathcal{E}_{1} \cdots \mathcal{E}_{n}$ have an $s^{\prime}=(s / n)$-section property. Since they and their Minkowski sums are centrally symmetric convex bodies, we can say even more: for any $k$-set $\alpha \subseteq\{1 \cdots n\}$ the body $\sum_{i \in \alpha} \mathcal{E}_{i}$ has a $k$-dimensional section by a linear subspace containing $s^{\prime} \cdot B_{k}$.

Observe also that, by monotonicity of mixed volume, $V\left(\mathcal{E}_{1} \cdots \mathcal{E}_{n}\right) \leq V\left(K_{1} \cdots K_{n}\right)$.

Let $\mathcal{E}_{i}=\left\{x:\left\langle A_{i} x, x\right\rangle \leq 1\right\}$, where $A_{1} \cdots A_{n}$ are positive definite matrices. We will show that the matrices $A_{i}^{-1}$ satisfy the conditions of Theorem 1.14 with $r_{1}=\cdots=r_{n}=$ $s^{2} / n^{4}$. We start with a simple lemma.

Lemma 5.1. For any $x \in \sum_{i \in \alpha} \mathcal{E}_{i}$ it holds that

$$
\left\langle\left(\sum_{i \in \alpha} A_{i}^{-1}\right)^{-1} x, x\right\rangle \leq|\alpha|^{2} .
$$


Proof. Write $x=\sum_{i \in \alpha} y_{i}$, where $\left\langle A_{i} y_{i}, y_{i}\right\rangle \leq 1$. Let $C=C_{\alpha}=\left(\sum_{i \in \alpha} A_{i}^{-1}\right)^{-1}$. Then $C$ is positive semidefinite and $C \preceq A_{i}$ for all $i \in \alpha$. It follows that

$$
\begin{aligned}
\langle C x, x\rangle & =\left\langle C\left(\sum_{i \in \alpha} y_{i}\right), \sum_{i \in \alpha} y_{i}\right\rangle=\sum_{i}\left\langle C y_{i}, y_{i}\right\rangle+\sum_{i \neq j}\left\langle C y_{i}, y_{j}\right\rangle \\
& \leq \sum_{i}\left\langle C y_{i}, y_{i}\right\rangle+\sum_{i \neq j}\left\langle C y_{i}, y_{i}\right\rangle^{1 / 2}\left\langle C y_{j}, y_{j}\right\rangle^{1 / 2} \\
& \leq \sum_{i}\left\langle A_{i} y_{i}, y_{i}\right\rangle+\sum_{i \neq j}\left\langle A_{i} y_{i}, y_{i}\right\rangle^{1 / 2}\left\langle A_{j} y_{j}, y_{j}\right\rangle^{1 / 2} \leq|\alpha|^{2} .
\end{aligned}
$$

Fix $\alpha \subseteq\{1 \cdots n\}$. Let $U$ be an $|\alpha|$-dimensional subspace $\mathbf{R}^{n}$ such that for any $x \in U$ with $\|x\| \leq s^{\prime}$ it holds that $\left\langle C_{\alpha} x, x\right\rangle \leq|\alpha|^{2}$. By the Courant-Fischer theorem, $C_{\alpha}$ has at least $|\alpha|$ eigenvalues $\leq|\alpha|^{2} /\left(s^{\prime}\right)^{2} \leq n^{4} / s^{2}$, and the matrix $\sum_{i \in \alpha} A_{i}^{-1}=C_{\alpha}^{-1}$ has at least $|\alpha|$ eigenvalues $\geq s^{2} / n^{4}$. Consequently, the matrices $A_{i}^{-1}$ satisfy the conditions of Theorem 1.14. Applying the theorem,

$$
D\left(A_{1}^{-1} \cdots A_{n}^{-1}\right) \geq \frac{s^{2 n}}{n^{4 n}} \cdot \frac{n !}{n^{n}} .
$$

Therefore, by (2),

$$
V\left(K_{1} \cdots K_{n}\right) \geq V\left(\mathcal{E}_{1} \cdots \mathcal{E}_{n}\right) \geq 3^{-(n+1) / 2} v_{n} D^{1 / 2}\left(A_{1}^{-1} \cdots A_{n}^{-1}\right) \geq\left(\Omega\left(s n^{-5 / 2}\right)\right)^{n} .
$$

In the last inequality we have used the fact that the volume $v_{n}$ of the $n$-dimensional unit ball is $(1 / \sqrt{\pi n})(2 \pi e / n)^{n / 2}\left(1+O\left(n^{-1}\right)\right)$.

\subsection{Proof of Theorem 1.16}

Proof. For a $k$-set $\alpha \subseteq\{1 \cdots n\}$, let $v_{1}(\alpha) \cdots v_{k}(\alpha)$ be vectors in $\bigcup_{i \in \alpha} U_{i}$ with $\operatorname{Vol}_{k}\left(\left[v_{1} \cdots v_{k}\right]\right) \geq \varepsilon^{k}$. Let $V=\bigcup_{\alpha \subseteq\{1 \cdots n\}}\left\{v_{1}(\alpha) \cdots v_{k}(\alpha)\right\}$. For $1 \leq i \leq n$, let $A_{i}=\sum_{v \in U_{i} \cap V} v \otimes v$. We will show that the matrices $A_{i}$ satisfy the conditions of Theorem 1.14 with $r_{k}=\left(\ell^{2} / 2\right) \cdot(\varepsilon / \ell)^{2 k}$.

Indeed, let $\alpha \subseteq\{1 \cdots n\}$, with $|\alpha|=k$. Let $B_{\alpha}=\sum_{j=1}^{k} v_{j}(\alpha) \otimes v_{j}(\alpha)$. Then

$$
A_{\alpha}=\sum_{v \in V \cap \cup_{i \in \alpha} U_{i}} v \otimes v \succeq \sum_{j=1}^{k} v_{j}(\alpha) \otimes v_{j}(\alpha)=B_{\alpha} .
$$

Therefore, it is sufficient to show that $B=B_{\alpha}$ has at least $k$ eigenvalues greater than or equal to $r_{k}$.

Let $X=\operatorname{Span}\left(v_{1}(\alpha) \cdots v_{k}(\alpha)\right)$. By the Courant-Fischer theorem it is enough to consider the $k \times k$ matrix $B \mid X$. We need a simple lemma:

Lemma 5.2. Let $v_{1} \cdots v_{k}$ be a basis of $\mathbf{R}^{k}$, and let $C=v_{1} \otimes v_{1}+\cdots+v_{k} \otimes v_{k}$. Then

$$
\operatorname{det} C=\operatorname{Vol}^{2}\left(\left[v_{1} \cdots v_{k}\right]\right) \text {. }
$$


Proof. The statement is immediate if $v_{1} \cdots v_{k}$ are orthogonal. If not, let $T$ be a matrix such that $T v_{1} \cdots T v_{k}$ are orthogonal, and consider $T C T^{t}=\sum_{j=1}^{k} T v_{j} \otimes T v_{j}$.

Therefore, $\operatorname{det}(B \mid X) \geq \varepsilon^{2 k}$. Next, consider the trace of $B \mid X$. We have $\operatorname{Tr}(B \mid X)=$ $\sum_{j=1}^{k} \operatorname{Tr}\left(v_{j} \otimes v_{j}\right)=\sum_{j=1}^{k}\left\|v_{j}\right\|^{2} \leq k \ell^{2}$. Let $\lambda_{1} \geq \lambda_{2} \cdots \lambda_{k}>0$ be the eigenvalues of $B \mid X$. They satisfy

$$
\prod_{i=1}^{k} \lambda_{i} \geq \varepsilon^{2 k}, \quad \sum_{i=1}^{k} \lambda_{i} \leq k \ell^{2}
$$

Lemma 5.3. Let $\lambda_{1} \geq \lambda_{2} \geq \cdots \geq \lambda_{k}$ be positive numbers satisfying (20). Then $\lambda_{k} \geq\left(\ell^{2} / 2\right) \cdot(\varepsilon / \ell)^{2 k}$.

Proof. For $k=1$ the claim is trivial. Assume $k \geq 2$. Let $\lambda_{k}=\delta$. Then $\prod_{i=1}^{k-1} \lambda_{i} \geq \varepsilon^{2 k} / \delta$ and $\sum_{i=1}^{k-1} \lambda_{i} \leq \leq k \ell^{2}-\delta$. By the arithmetic-geometric mean inequality applied to $\lambda_{1} \cdots \lambda_{k-1}$,

$$
\varepsilon^{2 k} \leq \delta \cdot\left(\frac{k \ell^{2}-\delta}{k-1}\right)^{k-1}
$$

Consider the function $f(x)=x \cdot\left(\left(k \ell^{2}-x\right) /(k-1)\right)^{k-1}$ on the interval $\left[0, k \ell^{2}\right]$. This function is 0 at 0 and it increases from 0 until its maximum at $x=\ell^{2}$. Observe that, by definition, $\varepsilon \leq \ell$, and therefore the point $\left(\ell^{2} / 2\right) \cdot(\varepsilon / \ell)^{2 k}$ is in the interval $\left[0, \ell^{2}\right]$ on which the function is increasing. Consequently, in order to prove the lemma, it is sufficient to check that $f\left(\left(\ell^{2} / 2\right) \cdot(\varepsilon / \ell)^{2 k}\right)<\varepsilon^{2 k}$. This inequality easily reduces to $2(k-1) \geq k-\frac{1}{2} \cdot(\varepsilon / \ell)^{2 k}$, which is, of course true.

Now, we apply Theorem 1.14:

$$
\begin{aligned}
\left(\frac{\ell^{2}}{2 e}\right)^{n} \cdot\left(\frac{\varepsilon}{\ell}\right)^{n(n+1)} & \leq \frac{n !}{n^{n}} \cdot \prod_{k=1}^{n} r_{k} \leq D\left(A_{1} \cdots A_{n}\right) \\
& =D\left(\sum_{v_{1} \in U_{1} \cap V} v_{1} \otimes v_{1} \cdots \sum_{v_{n} \in U_{n} \cap V} v_{n} \otimes v_{n}\right) \\
& =\sum_{v_{1} \cdots v_{n}} D\left(v_{1} \otimes v_{1} \cdots v_{n} \otimes v_{n}\right)=\sum_{v_{1} \cdots v_{n}} \operatorname{Vol}^{2}\left(\left[v_{1} \cdots v_{n}\right]\right) .
\end{aligned}
$$

The penultimate equality is based on multilinearity of the mixed discriminant. To see the last equality, observe that definition (1) together with Lemma 5.2 imply $D\left(v_{1} \otimes\right.$ $\left.v_{1} \cdots v_{n} \otimes v_{n}\right)=\operatorname{det}\left(v_{1} \otimes v_{1}+\cdots+v_{n} \otimes v_{n}\right)=\operatorname{Vol}^{2}\left(\left[v_{1} \cdots v_{n}\right]\right)$.

Since $|V| \leq n 2^{n-1}$, the number of sequences $v_{1} \cdots v_{n}$ is at most $n^{n} 2^{n(n-1)}$. It follows that there is a choice of vectors $v_{1} \cdots v_{n}$ with $v_{i} \in U_{i} \cap V$ such that

$$
\operatorname{Vol}\left(\left[v_{1} \cdots v_{n}\right]\right) \geq\left(\frac{1}{e^{1 / 2} n}\right)^{n / 2} \cdot 2^{-n^{2} / 2} \cdot\left(\frac{\varepsilon}{\ell}\right)^{n(n+1) / 2} \cdot \ell^{n}
$$




\section{References}

1. A. Aleksandrov, On the theory of mixed volumes of convex bodies, IV, Mixed discriminants and mixed volumes, Mat. Sb. (N.S.), 3 (1938), 227-251 (in Russian).

2. M. Avriel, Nonlinear Programming: Analysis and Methods, Prentice-Hall, Englewood Cliffs, NJ, 1976.

3. R. B. Bapat, Mixed discriminants of positive semidefinite matrices, Linear Algebra Appl., 126, 107-124, 1989.

4. R. B. Bapat and T. E. S. Raghavan, Nonnegative Matrices and Applications, Cambridge University Press, Cambridge, 1997.

5. I. Bárány and Z. Furedi, Computing the volume is difficult, Discrete Comput. Geom., 2 (1987), 319-326.

6. A. I. Barvinok, Computing mixed discriminants, mixed volumes, and permanents, Discrete Comput. Geom., 18 (1997), 205-237.

7. A. I. Barvinok, Polynomial time algorithms to approximate permanents and mixed discriminants within a simply exponential factor, Random Struct. Algorithms, 14 (1999), 29-61.

8. M. Dyer and A. Frieze, The complexity of computing the volume of a polyhedron, SIAM J. Comput., 17 (1988), 967-994.

9. M. Dyer, P. Gritzmann and A. Hufnagel, On the complexity of computing mixed volumes, SIAM J. Comput., 27(2) (1998), 356-400.

10. J. Edmonds, Submodular functions, matroids, and certain polyhedra, in Combinatorial Structures and Their Applications (R. Guy, H. Hanani, N. Sauer and J. Schönheim, eds.), Gordon and Breach, New York, 1970, pp. 69-87.

11. G. P. Egorychev, The solution of van der Waerden's problem for permanents, Adv. in Math., 42 (1981), 299-305.

12. D. I. Falikman, Proof of the van der Waerden's conjecture on the permanent of a doubly stochastic matrix, Mat. Zametki, 29(6) (1981), 931-938, 957 (in Russian).

13. S. Friedland, A lower bound for the permanent of a doubly stochastic matrix, Ann. of Math., 110 (1979), 167-176.

14. C. D. Godsil, Algebraic Combinatorics, Chapman and Hall, London, 1993.

15. M. Grötschel, L. Lovasz and A. Schrijver, Geometric Algorithms and Combinatorial Optimization, Springer-Verlag, Berlin, 1988.

16. L. Gurvits, Van der Waerden conjecture for mixed discriminants, Adv. in Math., to appear.

17. M. Jerrum and A. Sinclair, Approximating the permanent, SIAM J. Comput., 18 (1989), 1149-1178.

18. M. Jerrum, A. Sinclair and E. Vigoda, A polynomial-time approximation algorithm for the permanent of a matrix with non-negative entries, Proc. 33rd ACM Symp. on Theory of Computing, 2001.

19. F. John, Extremum problems with inequalities as subsidiary conditions, in Studies and Essays, Presented to R. Courant on His 60th Birthday, Interscience, New York, 1948.

20. B. Kalantari and L. Khachian, On the complexity of nonnegative matrix scaling, Linear Algebra Appl., 240 (1996), 87-104.

21. N. Linial, A. Samorodnitsky and A. Wigderson, A deterministic strongly polynomial algorithm for matrix scaling and approximate permanents, Proc. 30th ACM Symp. on Theory of Computing, 1998.

22. A. Nemirovski and U. Rothblum, On complexity of matrix scaling, Linear Algebra Appl. 302/303 (1999), 435-460.

23. Y. Nesterov and A. Nemirovskii, Interior-Point Polynomial Algorithms in Convex Programming, SIAM, Philadelphia, PA, 1994.

24. A. Panov, On mixed discriminants connected with positive semidefinite quadratic forms, Soviet Math. Dokl., 31 (1985).

25. R. Schneider, Convex Bodies: The Brunn-Minkowski Theory, Encyclopedia of Mathematics and Its Applications, vol. 44, Cambridge University Press, New York, 1993.

26. L. G. Valiant, The complexity of computing the permanent, Theoret. Comput. Sci., 8(2) (1979), 189-201.

Received April 17, 2000, and in revised form November 2, 2001. Online publication March 27, 2002. 